\title{
Quelques faits marquants de la dynamique récente des échanges de produits alimentaires
}

Benoît Daviron

\begin{abstract}
Some prominent facts of the recent dynamic of the international food trade

The renewal of international food trade, recorded since 1989, comes along with a considerable alteration of his compound and geography. Grains are loosing their central position for the benefit of animal proteins (meat and fish), fruits and vegetables. Import demand is gradualy rocking to Asia while a tripoly, opposing United States, EU and developping Asia, is emerging. This upsetting of the hierarchy of the countries comes along with a stronger regional trade in Asia and America, and, on the contrary, weaker internal relations in Eurafrica.
\end{abstract}

\section{Résumé}

Le renouveau des échanges internationaux de produits alimentaires, observé depuis 1989, s'accompagne d'importantes modifications de leur composition et de leur géographie. Les "grains" perdent la place centrale qu'ils occupaient dans les échanges depuis l'après-guerre au profit des protéines animales (viandes et poissons) et des fruits et légumes. La demande d'importation bascule peu à peu vers l'Asie tandis qu'émerge un triopole opposant les États-Unis, l'Ue et l'Asie en développement. Ce bouleversement de la hiérarchie entre les pays, s'accompagne d'un renforcement des échanges régionaux en Asie et en Amérique, et au contraire d'un affaiblissement des relations au sein de l'ensemble Eurafrique.

\section{Citer ce document / Cite this document :}

Daviron Benoît. Quelques faits marquants de la dynamique récente des échanges de produits alimentaires. In: Économie rurale. $\mathrm{N}^{\circ} 234-235,1996$. Globalisation des économies agricoles et alimentaires. Situation et prospective. pp. 10-16;

doi : https://doi.org/10.3406/ecoru.1996.4797

https://www.persee.fr/doc/ecoru_0013-0559_1996_num_234_1_4797

Fichier pdf généré le 08/05/2018 


\section{uelques faits marquants de la dynamique récente des échanges de produits alimentaires}

Ce texte tente de rendre compte des transformations récentes qu'ont connues les échanges internationaux de produits alimentaires (1). Depuis 1989, et après plusieurs années de crise, les échanges de produits alimentaires semblent avoir retrouvé le chemin de la croissance (entre 1989 et 1994 leur volume a augmenté de 3,5\% par an et leur valeur est passée de 204 à 267 milliards de dollars).

Sans prétendre fournir une analyse complète ni même donner une vision exhaustive de ce renouveau de la croissance des échanges, ce texte, très descriptif, tente d'en présenter les faits dominants. Il est organisé en trois partie. La première décrit les transformations de la composition des échanges en terme de produit. La seconde rend compte de l'évolution de la géographie des importations et des exportations. Enfin la dernière fournit quelques indications sur la dynamique des échanges régionaux.

Avant d'aller plus loin, précisons que nous avons exclu les échanges intracommunautaires des échanges internationaux. Toute analyse des échanges internationaux de produits alimentaires se heurte à la difficulté de leur traitement. Les élargissements successifs de l'UE et leur dynamisme propre ont fait passer leur part dans les échanges internationaux d'environ $5 \%$ au début des années 1960, à $15 \%$ au début des années 1970, $20 \%$ au début des années 1980 et $33 \%$ au début des années 1990 ! Quel statut faut-il leur donner? Le débat n'est guère facile à trancher. Pour notre part, nous avons pris le parti de les traiter comme des échanges nationaux, position qui nous semble la seule en mesure de rendre compte de la réalité des forces en présence dans les échanges mondiaux de produits alimentaires.

1. Le terme "produits alimentaires" recouvre dans ce texte, l'ensemble des produits inclus dans les divisions 0 (aliments et animaux vivants), 1 (boissons et tabacs), 22 (graines oléagineuses) et 4 (huiles végétales et animales) de la classification CTCI. Nous avons donc inclus dans l'analyse les poissons et produits de la mer mais en revanche exclu les matières premières agricoles (cuir et peaux, caoutchouc naturel et fibres textiles). II faut noter que les matières premières représentent une part fortement décroissante des échanges agricoles (10\% au début des années 1990 contre $23 \%$ au début des années 1960).

\section{Composition des échanges : la remise en cause de la place centrale des "grains"}

Les différents groupes de produits alimentaires ont connu des évolutions fortement différenciées depuis le début des années 1980. On peut ainsi placer à une extrémité la viande de volaille ou les fruits dont les échanges ont plus que doublé entre 1980 et 1993, et à l'autre extrémité les céréales, les graines oléagineuses ou le sucre dont les échanges stagnent résolument.

Tableau 1. Taux de croissance annuelle de la production et des échanges mondiaux en volume, 1980-1993

\section{Illustration non autorisée à la diffusion}

\section{Source : D'après FAO, USDA, Oil World, ITC diverses années.}

Ces évolutions différenciées s'expliquent partiellement par l'évolution des modèles de consommation. Croissance des revenus et changements culturels œuvrent clairement en faveur des fruits, de la viande et des produits de la mer. Les différences dans les taux de croissance des échanges ne font que refléter, pour partie, les écarts dans les taux de croissance de la production et de la consommation mondiale (2).

2. Comme le montre l'échantillon de produits du tableau 1 , il y a une relation globalement positive entre le taux de croissance de la production et le taux de croissance des échanges. Néanmoins, le sens de la relation de causalité n'a rien d'univoque. 
Les échanges ne sont cependant pas mus par les seules dynamiques de production et de consommation. Certains produits connaissent une internationalisation rapide de leur activité tandis que d'autres au contraire tendent à se "nationaliser" ou "re-nationaliser".

Paradoxe d'une période souvent présentée comme celle de l'internationalisation des activités économiques, la part de la production mondiale participant aux échanges est en recul pour le blé (elle passe de $21 \%$ en 1981-1983 à $19 \%$ en 1991-1993), les céréales secondaires (de $13 \%$ à $11 \%$ ), les graines oléagineuses (de $21 \%$ à $17 \%$ ), le sucre (de $29 \%$ à $26 \%$ ) ou le thé (de $39 \%$ à $36 \%$ ).

A l'inverse, les produits de la mer et la viande de volaille connaissent une internationalisation rapide. Comme le montre l'excellente étude de R. Henry et G. Rothwell (1995) le marché de la viande de volaille présente toutes les caractéristiques susceptibles d'en faire l'archétype de la dynamique actuelle des échanges de produits alimentaires: importance du débouché asiatique en pleine expansion, concurrence à l'exportation entre l'uE (France, Hollande), les États-Unis - devenus le premier exportateur en quelques années - et l'Asie en développement (la Thaïlande déjà déplacée par la Chine), rôle moteur des entreprises géantes et de leurs investissements.

L'idée d'une renationalisation des "grains" doit donc être immédiatement relativisée. Les céréales ou les graines oléagineuses, qui restent dans les pays, réapparaissent aussitôt dans les échanges sous forme de viande, de poissons et produits de la mer et, dans une moindre mesure, de tourteaux et d'huile. Une des questions essentielles de la période concerne ainsi les place respectives des "grains" (céréales et oléagineux) et des protéines animales (viandes et poissons) dans les échanges internationaux. La désintégration verticale à l'échelle mondiale entre production de "grains" et production de viande, qui était l'un des principaux moteurs des échanges de l'aprèsguerre et le fondement de la "domination" américaine sur ces échanges, semble aujourd'hui remise en cause.

L'examen de la structure des échanges internationaux de produits alimentaires confirme ces évolutions: forte diminution de la part des céréales, des graines oléagineuses, du sucre et des boissons tropicales (1), montée remarquable des poissons et produits de la mer, des fruits et légumes, de la viande et accessoirement du tabac. Ainsi, aujourd'hui, les fruits et légumes, et bientôt

1. La chute brutale de la part des boissons tropicales est, à l'exception du thé, plus dûe à l'effondrement des cours qu'à un ralentissement des échanges. Dans ce mouvement les boissons tropicales ont retrouvé leur part - des échanges alimentaires - de l'avant-guerre. II s'agit en quelque sorte d'une normalisation du statut des boissons tropicales liée à la crise des dispositifs de régulation des marchés mis en place dans les années 1960 (B.Daviron, 1995) les produits de la mer, font jeu égal avec les céréales tandis que le tabac dépasse l'ensemble des boissons tropicales !

Tableau 2. Composition des échanges mondiaux (hors-intra UE) de produits alimentaires

\section{Illustration non autorisée à la diffusion}

\section{Géographie des importations : le basculement asiatique}

L'ensemble parfois appelé Eurafrique, cette partie du monde que nous avons l'habitude de voir au centre des planisphères et qui constituait depuis "toujours" le cœur du marché des produits alimentaires, est en train de perdre sa place. En effet, l'Europe de l'Ouest, qui représentait à elle seule près de la moitié des débouchés jusqu'à la fin des années 1960, avait fortement reculé au cours de la décennie 1970 mais avait été relayée, à la faveur du boom pétrolier et de l'euphorie financière, par ses "périphéries" : Europe de l'Est, Proche Orient, Afrique. Au recul de la part de l'Europe de l'Ouest dans les importations mondiales avait correspondu la progression de la part des ces trois régions. Au début des années 1980, l'ensemble Eurafrique continuait ainsi de représenter $57 \%$ des importations mondiales.

Or, au cours des dix dernières années, si la part de l'Europe de l'Ouest demeure inchangée, en revanche celles de toutes ses périphéries reculent. Aujourd'hui l'Eurafrique ne représente plus "que" $46 \%$ des importations mondiales.

Dans le même mouvement, l'Asie émerge peu à peu comme le futur centre des marchés alimentaires. Prise dans son ensemble, la part de la région est passée de 20 à 
Tableau 3. Importations mondiales de produits alimentaires par pays ou région

Source : D'après CNUCED, FAO, OCDE diverses années.

$30 \%$ des importations mondiales entre 1981-1983 et 1991-1993, le Japon et l'Asie en développement faisant part égale.

Le Japon est désormais le deuxième pays importateur après l'UE et destiné, au rythme actuel de croissance de ses achats (1), à devenir le premier dans les prochaines années.

L'évolution de la structure des importations japonaises illustre très clairement les bouleversements que connaît actuellement la composition des échanges internationaux de produits alimentaires : effondrement de la part des "grains" - malgré la hausse des importations de riz - et hausse des protéines animales (viandes mais plus encore produits de la mer), des fruits et légumes et du groupe "boissons, tabac".

Tableau 4. Part des principaux produits dans les importations alimentaires du Japon

\section{Illustration non autorisée à la diffusion}

Source : D'après $F A O$, diverses années.

La croissance des importations de l'Asie en développement est tirée en premier lieu par les 4 dragons industriels de la région (Corée, Taïwan, Hongkong, Singapour) mais aussi par des pays considérés comme plus agricoles. L'évolution des importations de la Thaillande est de ce point de vue saisissante : elles ont été multipliées par 5 entre 1985 et 1994 !

1. D'après l'OMC (1995) les importations japonaises de produits alimentaires ont augmenté de $10 \%$ par an depuis 1990 et le Japon représentait en $199417 \%$. des importations mondiales.
Cette dynamique des importations de l'Asie en développement est d'autant plus remarquable que jusqu'à aujourd'hui les deux géants de la région (Chine et Inde) s'en sont maintenus relativement à l'écart. L'Inde demeure en effet totalement absente des marchés : la valeur de ses importations - en dollar courant - est équivalente à ce qu'elle était dans les années 1960. Les importations chinoises ont, elles, certes cru mais de manière modérée et surtout fortement instable $(+1 \%$ en $1992,-24 \%$ en $1993,+70 \%$ en 1994).

\section{Tableau 5. Évolution des importations alimentaires} de pays asiatiques en développement

\section{Illustration non autorisée à la diffusion}

Source : D'après OMC, 1995 et FAO diverses années.

\section{Géographie des exportations : vers un triopole?}

Tableau 6. Exportations mondiales de produits alimentaires par pays ou régions

\section{|llustration non autorisée à la diffusion}

Source : D'après CNUCED, FAO, OCOE diverses années.

\section{La reprise us}

La position des États-Unis s'est avérée extrêmement sensible à la conjoncture des marchés. En effet ceux-ci ont pris à leur charge - de bon ou de mauvais gré - une 
bonne partie des variations qu'a connu le commerce des produits alimentaires. Durant les années 1980 leur part de marché a connu tout d'abord une très forte chute - elle est passé entre 1981 et 1986 de $23 \%$ à $16 \%$ des exportations mondiales - puis une certaine reprise avec une stabilisation autour de $20 \%$ (voir graphique 1 ).

Cette reconquête relative opérée par les États-Unis depuis 1986 s'est appuyée sur une importante recomposition de la structure des exportations. La position dominante des États-Unis dans les échanges mondiaux de produits alimentaires s'appuyait en effet sur une très forte spécialisation dans l'exportation de grains (céréales, graines oléagineuses, tourteaux). Ces produits ont représenté jusqu'à $75 \%$ des exportations alimentaires du pays à la fin des années 1970. La décennie 1980 a totalement bouleversé ce schéma. Désormais les grains ne représentent plus que $39 \%$ des exportations et trois "produits" (viandes, fruits et légumes, tabac) ont conquis, à eux trois, une place équivalente.

\section{Tableau7. Part des principaux produits}

dans les exportations alimentaires des États-Unis

\section{Illustration non autorisée à la diffusion}

Source : dapres FAO, diverses annees

\section{La conquête de la parité par I'UE}

Pour l'UE, poursuivant sur sa lancée de conquête des marchés internationaux de produits alimentaires, les années 1980 datent l'accès à une situation de parité avec les États-Unis. L'évolution de la part des exportations de l'UE - hors échanges intracommunautaires - dans les échanges mondiaux fait clairement apparaître ce phénomène (voir graphique 1). Cette conquête de la parité coïncide très précisément avec l'ouverture des négociations de l'Uruguay Round.

\section{La percée asiatique}

La montée de l'UE ne doit cependant pas nous cacher la remarquable percée de l'Asie en développement. Cette région qui, à l'égal des autres régions en développement, subissait, jusqu'au début des années 1970, une marginalisation progressive dans les échanges, effectue depuis lors une rapide reconquête du marché. Ce mouvement s'est encore accéléré depuis le milieu des années 1980 laissant sur place les autres régions en développement. Au total, la région fait aujourd'hui jeu égal avec l'UE et les États-Unis dans les exportations mondiales de produits alimentaires
Quatre pays, qui réalisent $70 \%$ des exportations de l'Asie en développement, tirent les exportations de la région. La Chine, avec 12 milliard de $\$$ de ventes en 1994, arrive largement en tête, en troisième position au niveau mondial, et avec une part des exportations mondiales $(5 \%)$ désormais nettement supérieure à celle de l'Afrique sub-saharienne $(3 \%)$. Viennent derrière la Thaillande avec 9 milliard de $\$$, puis au coude à coude, Malaisie et Indonésie ( 5 milliards de \$).

C'est évidemment forcer le trait que de présenter aujourd'hui l'offre d'exportation mondiale comme un triopole. L'Asie en développement ne présente en aucun cas une unité d'action équivalente à celle des États-Unis ou de l'UE. Toutefois il n'est pas exclu que les prochaines années conduisent les pays exportateurs d'Asie à définir des positions communes voire des stratégies de commercialisation conjointes. Une telle évolution serait d'abord permise par le caractère régional d'un certain nombre de réseau d'opérateurs privés (réseaux chinois). Elle serait aussi permise par la mise en place de coordinations régionales dans le cadre de l'ASEAN, comme l'illustre la création récente du Club Asiatique de l'Huile Végétale (AVOC).

\section{Graphique 1. Exportations de produits alimentaires des États-Unis, de l'Union Européenne et de l'Extrême Orient, 1961-1994}

En \% du total des échanges mondiaux de produits alimentaires, hors intra-UE

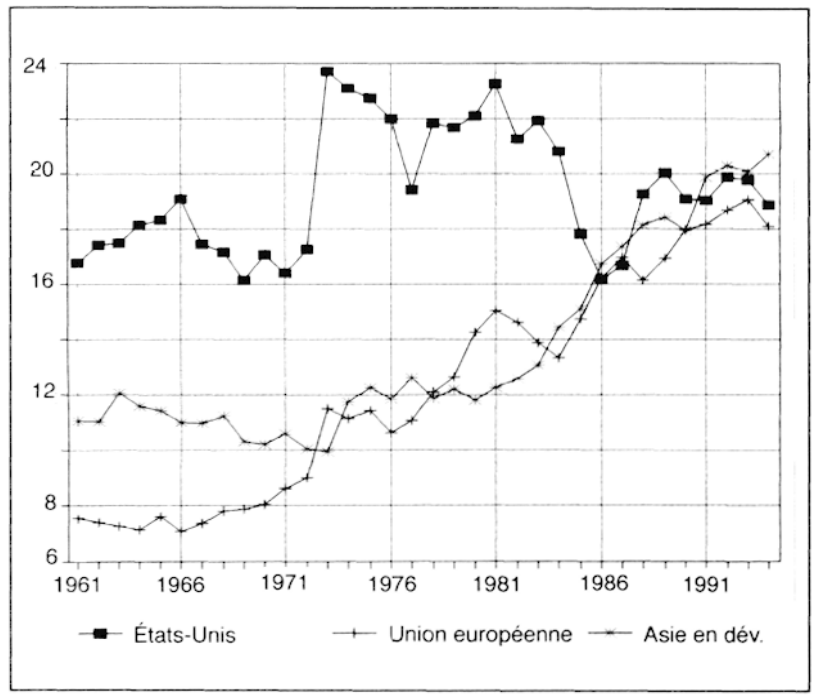

\section{Régionalisation versus mondialisation}

Nous avons tenté d'aborder ce point en distinguant trois grands ensembles : Eurafrique, Asie et Amérique. Nous avons comparé la géographie des échanges de ces ensembles en 1980 et 1994 (voir graphique 2) et complété l'analyse par l'étude des origines et des destinations des échanges des "pays" dominants de chaque ensemble (Japon, États-Unis, UE).

Nous aboutissons évidemment une vision très générale mais qui permet de dégager les mouvements dominants de la période. 


\section{Échanges intra-régionaux : trois dynamiques contrastées}

Globalement les échanges intra-régionaux sont en expansion. Leur part dans les échanges mondiaux est passée de $50 \%$ en 1980 à $57 \%$ en 1994. Ce chiffre global recouvre toutefois des évolutions fortement différenciées entre les trois grandes régions du monde.

L'Asie est à la tête du mouvement. L'importance des échanges intra-asiatiques est clairement croissante - leur part est passée de 12 à $17 \%$ des échanges mondiaux entre 1980 et 1994 - et ce mouvement s'appuie en tout premier lieu sur le très fort dynamisme des exportations de l'Asie en développement vers le Japon. La croissance rapide des importations japonaises joue en effet pleinement en faveur des exportateurs de la région: Asie du Sud-Est, mais aussi Chine, Taïwan ou Corée (produits de la mer oblige). La part de l'Asie en développement dans les importations du Japon est ainsi passée de $21 \%$ à $35 \%$.

Mais le dynamisme des échanges asiatiques repose aussi sur l'accroissement des échanges interne à l'Asie en développement dont les pays manifestent une claire préférence pour les importations d'origine régionale (voir tableau 8). Il faut souligner au passage la place croissante qu'occupe l'ensemble "Asie" dans les exportations des pays de l'Asie en développement.

La région "Amérique" connaît elle aussi un développement soutenu des échanges internes (ils sont passées de $12 \%$ à $16 \%$ des échanges mondiaux) mais elle offre un cas de figure quelque peut différent puisqu'ici la croissance des échanges est bi-centrée : au Nord autour des États-Unis, au Sud principalement autour du Mercosur.

Les échanges internes à l'Amérique du Nord (Mexique compris) ont été multipliés par 3 en valeur entre 1980 et 1994. Mais l'existence de l'ALENA ne doit pas faire illusion, les États-Unis sont au centre, incontestablement au centre, et l'ALENA n'est pour l'instant que la somme des relations bi-latérales qu'ils entretiennent avec leurs voisins. En 1994, face aux 13 milliards de dollars d'échanges Canada/États-Unis et aux 8 milliards d'échanges Mexique/États-Unis, les 400 millions d'échanges Mexique /Canada pèsent peu ! Le développement des échanges entre les États-Unis et ses voisins n'en est pas moins remarquable. Leurs part dans les importations et les exportations des États-Unis est passée respectivement de $19 \%$ à $29 \%$ et de $9 \%$ à $21 \%$.

Cet incontestable phénomène d'intégration "de proximité" s'accompagne d'une certaine désaffection vis-à-vis du reste du continent. En terme relatif, les États-Unis commercent de moins en moins avec l'Amérique du Sud et l'Amérique centrale. Du point de vue de la dynamique d'échange intra-américaine, cette évolution est compensée par un rapide développement des échanges entre pays latino-américain dans le cadre du Mercosur d'abord mais aussi entre tous les pays voisins (Argentine/Chili, Colombie/Venezuela... Voir l'article de Y. Chaloult et G. Hillcoat dans ce même numéro).

En résumé on peut dire que l'ensemble Amérique est actuellement mû par deux dynamiques d'intégration juxtaposées tendant à affaiblir les relations entre le Nord et le Sud du continent.

De son côté, l'ensemble Eurafrique semble poursuivre une dynamique à contre-courant des dynamiques américaines et asiatiques. En effet, entre 1980 et 1994 la part des échanges intra-Eurafrique dans les échanges mondiaux a diminué. Cette diminution, relative, est d'abord liée à l'effondrement - en valeur absolue - des échanges entre les pays d'Europe de l'Est. Disparition du СОМЕСОМ et crise économique se sont ici conjugués pour provoqué une contraction brutale du commerce. Mais elle s'explique aussi par le peu de dynamisme des relations qu'entretient l'Union Européenne avec ses périphéries, au contraire des États-Unis et du Japon.

Ce manque de dynamisme s'illustre tout d'abord par l'importante diminution de la part de l'Eurafrique dans les exportations de l'UE (de $70 \%$ à $58 \%$ ). Cette perte d'importance n'est pas liée à la concurrence de "l'extérieur" dans l'approvisionnement de l'Europe de l'Est, de l'Afrique ou du Moyen Orient, mais à la régression de leurs importations "toutes origines". De fait, la place de l'UE dans l'approvisionnement de ses périphéries s'est maintenue voire fortement accrue comme dans le cas de l'Europe de l'Est (de $12 \%$ à $42 \%$ ). La région Eurafrique se caractérise ainsi par un décalage marqué entre le développement de la capacité exportatrice de son centre - l'UE - et l'évolution du pouvoir d'achat (d'importation) des régions périphériques.

L'absence de dynamisme se retrouve aussi dans les exportations des périphéries vers l'UE. Le poids de ses voisins stagne dans les importations de l'UE (35\%). Ce constat devrait être nuancé, il y a en effet un développement rapide des exportations d'un nombre limité de pays proche - Maroc et Turquie en sont de clairs illustrations - et déclin de la part des pays lointains. Il faut toutefois constater que globalement, l'UE est désormais le "pôle" de la Triade qui s'approvisionne le moins, en terme relatif, auprès de ses périphéries.

\section{Échanges inter-régionaux : contraction atlantique et rééquilibrage Euro-Asiatique}

L'évolution la plus marquante dans le domaine du commerce alimentaire entre les trois grands ensembles que nous avons distingués concerne les échanges transatlantiques qui ont subit une contraction d'ampleur du fait de la diminution des exportations de l'Amérique vers l'Eurafrique.

Cette contraction a affecté essentiellement la position des États-Unis sur le marché Eurafrique et plus encore sur le marché européen. La part des États-Unis dans les importations de l'UE est passée de $25 \%$ en 1980 à $13 \%$ 
en 1994 alors que dans le même temps la part de l'Amérique Latine passait de $22 \%$ à $30 \%$ s'imposant sans conteste comme le premier fournisseur de l'Europe.

Pour l'ensemble Amérique, ce recul - toujours relatif des exportations vers l'Eurafrique a été partiellement compensé par accroissement des exportations vers l'Asie. Les exportations de l'Amérique vers l'Asie sont désormais équivalentes à celles de l'Amérique vers Eurafrique alors qu'elles n'en représentaient que la moitié en 1980. La destination des exportations des États-Unis a ainsi totalement changé. La part de l'Europe de l'Ouest est passée de $30 \%$ à $19 \%$ des exportations tandis que la part du Japon passait de $15 \%$ à $25 \%$.

\section{Graphique 2 : Échanges de produits alimentaires} des trois grands ensembles 1980 et 1994

En \% du total des échanges mondiaux hors intra-UE

\section{0}

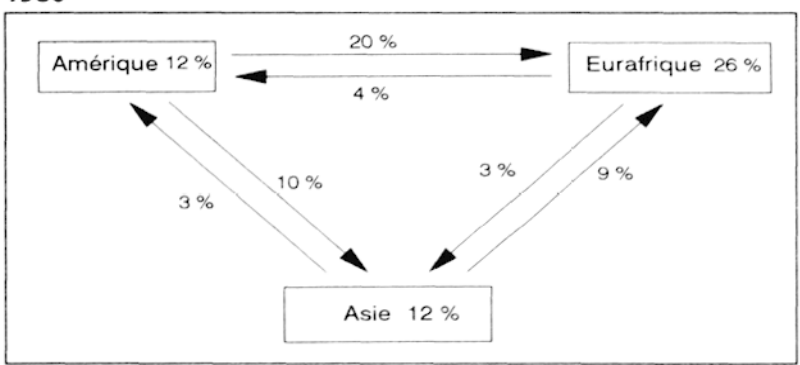

1994

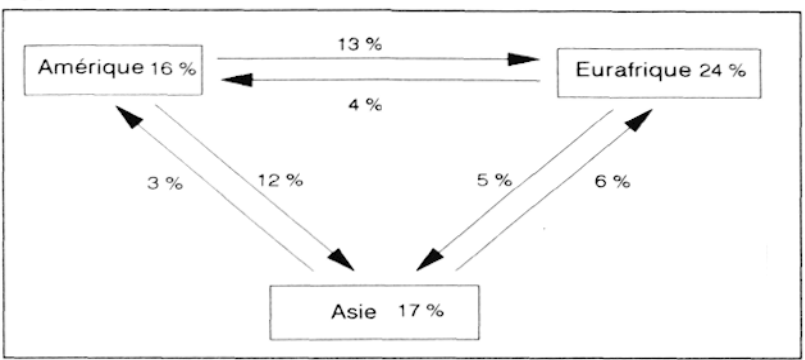

Les chiffres inscrits dans les carrés indiquent la part des échanges internes à chaque ensemble dans les échanges mondiaux.

Le rééquilibrage des échanges entre l'Eurafrique et l'Asie est la deuxième évolution caractéristique de la période. En 1980, l'Eurafrique enregistrait, dans ses échanges alimentaires, un déficit important vis à vis de l'Asie. L'Europe de l'Ouest a elle seule importait de l'Asie trois fois plus qu'elle n'y exportait. De même, l'Europe de l'Est et le Proche Orient s'approvisionnaient de manière importante en produits asiatiques. Là encore la situation a radicalement changé. La contraction des débouchés proche-orientaux et de l'Europe de l'Est, la faible croissance des importations européennes ont d'abord conduit aux déclins relatifs des exportations de l'Asie vers l'ensemble Eurafrique. Par ailleurs l'UE et dans une bien moindre mesure l'Europe de l'Est et l'Afrique ont bénéficié d'une partie de la croissance de la demande d'importations asiatique. Pour la première fois en 1994, l'Europe a enregistrer un excédent dans ses échanges de produits alimentaires avec l'Asie.

Le recul de la part, dans les échanges mondiaux, des exportations asiatiques "vers l'extérieur" est la dernière évolution que nous voudrions relever. Malgré la croissance de la production alimentaire asiatique, malgré aussi la croissance des exportations, le dynamisme de la demande régionale interdit désormais à l'Asie la conquête de marchés extérieurs. Depuis 1986, la croissance de la part de marché de l'Asie en développement se fait exclusivement par des exportations vers la région. Plus encore, l'Asie, prise dans son ensemble, enregistre un déficit croissant vis à vis du reste du monde. Au contraire de ce qu'avait pu laissé penser l'évolution de certains marchés particuliers (riz, cacao), l'Asie n'est pas près venir noyer le monde sous ses exportations alimentaires.

\section{Conclusion}

Le renouveau de la croissance des échanges de produits alimentaires constaté depuis 1989 s'accompagne de nombreuses modifications de sa composition comme de sa géographie. De biens plus amples travaux seraient nécessaires pour mieux préciser les évolutions en cours et surtout pour les interpréter. A ce stade nous voudrions en conclusion souligner trois points : 
1. Il faut cesser d'avoir une "lecture céréalière" des échanges alimentaires mondiaux. La littérature sur les échanges internationaux de produits alimentaires a longtemps pris comme objet d'étude et référence exclusive les marchés des "grains". Cette démarche est de moins en moins justifiée du fait tout d'abord de la perte d'importance de ce groupe de produit. En outre, elle conduit dans bien des cas à une vision récessive de la dynamique des échanges. Les marchés céréaliers et des graines oléagineuses stagnent certes, et ne sont pas les seuls, mais bien d'autres marchés connaissent une croissance comparable à celle des produits manufacturés. Enfin, cette focalisation sur les marchés des "grains" conduit à une représentation déformée de la structure des marchés de produits alimentaires et de la place des États. En effet, les grains sont par excellence le domaine d'intervention privilégié des États. Or, l'évolution de la composition des échanges avec la montée des produits à État minimum (fruits et légumes, produits de la mer) favorise un rééquilibrage en faveur du "privé" avant même toute libéralisation organisée par l'OMC.

2. La notion de globalisation renvoie à la dimension spatiale du développement économique mondial. Nation, région ou monde, quel est aujourd'hui l'espace pertinent ou fonctionnel de circulation, des produits et des facteurs, mais aussi de coordination et de régulation? Sans évidemment trancher le débat, l'étude de l'évolution des échanges souligne deux tendances indiquant une recomposition des espaces économiques :

- du point de vue général, la dynamique forte des échanges de proximité au moins pour les États-Unis (Mexique, Canada) et l'ue (Maroc, Turquie) qui s'accompagne d'un affaiblissement des relations avec les périphéries lointaines ;

- les évolutions inverses de la place des "grains" et de viandes dans les échanges mondiaux et plus encore dans celle des exportations des États-Unis, qui révèlent une réintégration spatiale (au moins au niveau national) de la "filière viande" et remettent en cause un des rares exemples de division internationale du travail à l'intérieur du secteur agricole.

Ces deux tendances sont conformes à certaines thèses relatives aux caractéristiques spatiales des nouveaux modèles d'organisation des relations intra et inter-entreprises (voir Ch. Oman, 1994 ; G. Benko et A. Liepietz, 1992 ; A. Rallet et A. Torre, 1995) qui souligne la nécessité d'un rapprochement physique des acteurs d'une même filière. Jusqu'où peut-on pousser cette hypothèse pour le secteur agro-alimentaire?

3. L'Asie toujours l'Asie. L'Asie exporte de plus en plus, l'Asie importe de plus en plus, l'Asie commerce de plus en plus et d'abord avec elle même. Cette montée des échanges asiatiques semble inéluctable et évidente au regard de la démographie. Comment toutefois ne pas opposer le dynamisme asiatique avec l'atonie Eurafricaine? Avec le Japon la dynamique de la demande d'importation et celle de l'investissement se conjuguent pour impulser la croissance de la production et des exportations de l'Asie et au delà, un développement soutenu des économies de la région. Au regard de cette évolution l'Europe semble quelque peu "marcher sur la tête". Fondée sur un projet visant à la sécurité alimentaire, l'UE se trouve engagée dans une course à l'exportation qui ne laisse que bien peu de place à l'organisation d'une division du travail favorables à ses voisins. Le développement des "périphéries européennes" pourra-t-il vraiment se faire sans une croissance de leurs exportations agricoles et alimentaires... vers l'Union européenne?

Benoît DAVIRON • CIRAD.

Juin 1996.

\section{RÉFÉRENCES BIBLIOGRAPHIQUES}

Benko G. et Liepietz A. (ed). Les régions qui gagnent. Le Seuil, Paris, 1992, 424 p.

CNUCED - Conférence des Nations-Unies sur le Commerce et le Développement. Manuel de statistiques du commerce et du développement. Genève, diverses années.

Daviron B. Le pire n'est jamais certain : élément sur la dynamique des marchés internationaux de produits tropicaux. In, S. Calabre (ed.) Matières premières, marchés mondiaux, déséquilibres, organisation. Economica, Paris, 1995, pp. 65-93.

FAO - Faostat. Base de donnée informatique.

Henry R., Rothwell G. The world poultry industry. Washington, Ifc, 1995, $74 \mathrm{p}$.

Mielke T. Oil World 2012. Hambourg, Ista, 1994.
OCDE. Statistiques du commerce extérieur. Paris, diverses années.

Oman Ch. Globalisation et régionalisation : quels enjeux pour les pays en développement? Centre de développement de l'OCDE, Paris, 1994, 145 p.

Organisation mondiale du commerce. Le commerce international, tendances et statistiques, 1995. OMC, Genève, 1995, $178 \mathrm{p}$.

Rallet A., Torre A. (ed). Economie industrielle et économie spatiale. Economica, Paris, 1995, 473 p.

USDA - United State Department of Agriculture. Grain : world market en trade. Divers numéros. 